\title{
Relationship of Red Cell Index with the Severity of Chronic Obstructive Pulmonary Disease
}

This article was published in the following Dove Press journal:

International Journal of Chronic Obstructive Pulmonary Disease

\author{
Yiben Huang $\mathbb{D}^{1, *}$ \\ Jianing Wang ${ }^{1,2, *}$ \\ Jiamin Shen ${ }^{1,2, *}$ \\ Jiedong $\mathrm{Ma}^{\mathrm{l}, 2}$ \\ Xiaqi Miao ${ }^{1,2}$ \\ Keke Ding ${ }^{1,2}$ \\ Bingqian Jiang ${ }^{1,2}$ \\ Binbin $\mathrm{Hu}^{\mathrm{I}, 2}$ \\ Fangyi $\mathrm{Fu}^{\mathrm{l}, 2}$ \\ Lingzhi Huang' \\ Meiying $\mathrm{Cao}^{3}$ \\ Xiaodiao Zhang (D) \\ 'Department of Respiratory Medicine, \\ The Third Affiliated Hospital of Wenzhou \\ Medical University, Wenzhou, People's \\ Republic of China; ${ }^{2}$ School of the First \\ Clinical Medical Sciences, Wenzhou \\ Medical University, Wenzhou, People's \\ Republic of China; ${ }^{3}$ Department of \\ Cardiology, The Third Affiliated Hospital \\ of Wenzhou Medical University, \\ Wenzhou, People's Republic of China
}

*These authors contributed equally to this work
Background: We aimed to investigate the association between red cell index $(\mathrm{RCI})$ and the severity of Chronic Obstructive Pulmonary Disease (COPD), and compare predictive value of RCI, neutrophil-lymphocyte ratio (NLR) and platelet-lymphocyte ratio (PLR) for the severity of COPD.

Methods: A total of 207 participants were recruited (100 COPD patients and 107 healthy controls). COPD patients were divided into two groups according to the optimal cut-off value of RCI determined by the receiver operating characteristic (ROC) curve. Pearson's correlation test, logistic regression analysis and other tests were performed.

Results: Compared with low RCI group, the forced expiration volume in 1 second $\left(\mathrm{FEV}_{1}\right)$ and $\mathrm{FEV}_{1}$ in percent of the predicted value $\left(\mathrm{FEV}_{1} \%\right)$ in high RCI group were lower $(p=$ $0.016, p=0.001)$. There was a negative correlation between RCI and $\mathrm{FEV}_{1} \%(r=-0.302$, $p=0.004$ ), while no correlation between $\mathrm{FEV}_{1} \%$ and NLR as well as PLR were found. RCI showed higher predictive value than NLR and PLR for predicting Global Initiative for Chronic Obstructive Lung Disease classification (GOLD), with a cut-off value of 1.75 and area under the curve (AUC) of $0.729(p=0.001)$. Multivariate logistic regression analysis proved that RCI was an independent factor for lung function in COPD patients (odds ratio $[\mathrm{OR}]=4.27,95 \% \mathrm{CI}: 1.57-11.63, p=0.004)$.

Conclusion: RCI is a novel biomarker that can better assess pulmonary function and severity of COPD than NLR and PLR. Higher RCI is related to deterioration of pulmonary function.

Keywords: COPD, pulmonary function, RCI, biomarker

\section{Introduction}

Chronic obstructive pulmonary disease (COPD) has always been a major public health problem, and is still a severe challenge in the 21 st century. At the same time, it has been attracting worldwide attention due to its high prevalence, morbidity and mortality. ${ }^{1}$ COPD is a common, preventable and curable disease characterized by progressive inflammation of the airways, alveoli and capillaries caused by exposure to harmful particles or gases, as well as the resulting emphysema and lung parenchyma peripheral loss, persistent respiratory symptoms and airflow limitation and other pathophysiological changes. ${ }^{2,3}$

Neutrophil-lymphocyte ratio (NLR) is considered as a reliable marker of disease severity and prognosis, as lymphocytes and neutrophils actively participate in the pathophysiological mechanism of emphysema and COPD. ${ }^{4,5}$ Besides, other studies have shown that platelet-lymphocyte ratio (PLR) is also an effective marker of inflammation in evaluating the severity of COPD patients. ${ }^{6}$ However, studies

\footnotetext{
Correspondence: Xiaodiao Zhang Department of Respiratory Medicine, The Third Affiliated Hospital of Wenzhou Medical University, No. 108 Wansong People's Republic of China Tel +86-577-65866223 Fax +86-577-65866586 Email xiaodiao_zhang@126.com

Meiying Cao

Department of Cardiology, The Third Affiliated Hospital of Wenzhou Medical University

Email519243591@qq.com
}

International Journal of Chronic Obstructive Pulmonary Disease 2021:16 825-834

825

DovePress if in $>$


also found that there was no difference between GOLD A-D groups and NLR as well as PLR. ${ }^{7}$

Relevant studies have shown that the lung function is related to the production of platelets (PLT). ${ }^{8}$ Moreover, Makhlouf et $\mathrm{al}^{9}$ observed that there was a significantly positive correlation between platelets and white blood cell (WBC). However, red blood cell (RBC) count and hemoglobin ( $\mathrm{Hb}$ ) were found to be inversely correlated with platelets. And RBC has also been found to be related to the diagnosis of COPD. ${ }^{8}$ The decrease in $\mathrm{Hb}$ level amplitude is related to oxygen intake. ${ }^{10}$ Rasmussen et $\mathrm{al}^{11}$ recently reported that low $\mathrm{Hb}$ was associated with an increasing 90-day mortality of admission to the intensive care unit (ICU) in COPD patients having acute respiratory failure. And higher levels of $\mathrm{Hb}$ were associated with a better long-term survival. ${ }^{12}$

Now, there is a new indicator red cell index (RCI), which is related to PLT, RBC, $\mathrm{Hb}$ and lymphocyte (Lym). RCI is calculated by the following equation: $(\mathrm{RBC} \times \mathrm{Hb}) /(\mathrm{Lym} \times$ PLT). ${ }^{13}$ In theory, RCI is inversely proportional to pulmonary function and it is a simple and effective indictor to evaluate pulmonary function, but no studies reported the potential value of RCI as a COPD biomarker. ${ }^{13}$

The purpose of our study was to evaluate the correlation between the new indicator RCI and the severity of COPD and compare RCI with NLR and PLR in predicting the severity of COPD.

\section{Patients and Methods Study Population and Data Collection}

From February 2018 to February 2019, we conducted a cross-sectional study in COPD patients and recruited 100 subjects diagnosed with COPD from the Department of Respiratory Medicine, the Third Affiliated Hospital of Wenzhou Medical University, as well as 107 age- and sexmatched cases served as healthy controls. The healthy controls were people purely healthy person, that is, without any disease. All our patients were admitted to the hospital for COPD and diagnosed by licensed physician.

Inclusion criteria were as follows: 1) age more than 40years old; 2) diagnosis of COPD as defined in the GOLD guidelines. ${ }^{14}$ The exclusion criteria were: 1 ) obstructive sleep apnea hypopnea syndrome; 2) alimentary tract hemorrhage; 3) renal insufficiency; 4) arrhythmia; 5) coronary atherosclerotic heart disease; 6) cardiac insufficiency; 7) liver cirrhosis; 8) alcoholic hepatitis; 9) hypothyroidism; 10) hyperthyroidism; 11) Hashimoto's thyroiditis (Figure 1). ${ }^{10,15-18}$
The study was approved by the Ethics Committee of the Third Affiliated Hospital of Wenzhou Medical University and conducted in accordance with the Declaration of Helsinki. All subjects had signed a written informed consent form.

Following recruitment of subjects was taken during their hospitalizations for COPD. Demographic information, medical history like age, gender, body mass index (BMI), smoking, duration of disease, and comorbidities were collected and analyzed. Blood cell-related indicators such as red blood cells, lymphocytes, platelets, neutrophils and hemoglobin were collected on 24 hours admission, and blood samples were also collected to analyze blood biochemistry and arterial blood gas. These tests were carried out using XT-1800i (Sysmex, Kobe, Japan). Parameters like RCI, NLR and PLR of COPD patients and healthy controls were calculated. RCI was calculated using the following equation: $(\mathrm{RBC} \times \mathrm{Hb}) /$ $\left(\right.$ Lym $\times$ PLT). ${ }^{13}$ The pulmonary function test was performed using spirometer (CareFusion, San Diego, California, USA) at least three times in the spirometry laboratory in a seated position according to the international guidelines. ${ }^{19}$ Forced expiratory volume in 1 second $\left(\mathrm{FEV}_{1}\right), \mathrm{FEV}_{1}$ in percent of the predicted value $\left(\mathrm{FEV}_{1} \%\right)$, forced vital capacity $(\mathrm{FVC})$ and $\mathrm{FEV}_{1} / \mathrm{FVC}$ of COPD patients were recorded as significant indicators of the severity of pulmonary function. ${ }^{20-22}$ Furthermore, we also collected scales of COPD patients within 7 days after hospital admission including BODE (BMI, airway obstruction, dyspnea, exercise capacity) ${ }^{23} \mathrm{ADO}$ (age, dyspnea and airflow obstruction) ${ }^{24}$ and DOSE (MRC Dyspnea Scale, airflow obstruction, smoking status and exacerbations). ${ }^{25}$

\section{Diagnostic Criteria of COPD}

Patients with COPD were categorized in severity grades I-IV using spirometry (GOLD I: $\mathrm{FEV}_{1} \geq 80 \%$ predicted; GOLD II: $50 \% \leq$ FEV $_{1}<80 \%$ predicted; GOLD III: $30 \% \leq$ FEV $_{1}<$ $50 \%$ predicted; GOLD IV: $\mathrm{FEV}_{1}<30 \%$ predicted). ${ }^{26}$

\section{Statistical Analysis}

All statistical analyses were performed using SPSS 25.0 (IBM Analytics). Mean \pm standard deviation was used to present continuous variables of normal distribution. The Chi-squared $(\chi 2)$ test or Fisher test was used for the comparison of categorical variables. We used the independent sample $t$ test to compare the differences of clinical characteristics between COPD patients and healthy controls, low RCI group and high RCI group. Relationships between RCI, NLR, PLR and $\mathrm{FEV}_{1} \%$ were evaluated by Pearson's correlation test. In order to estimate the value of RCI for predicting pulmonary function compared with the 


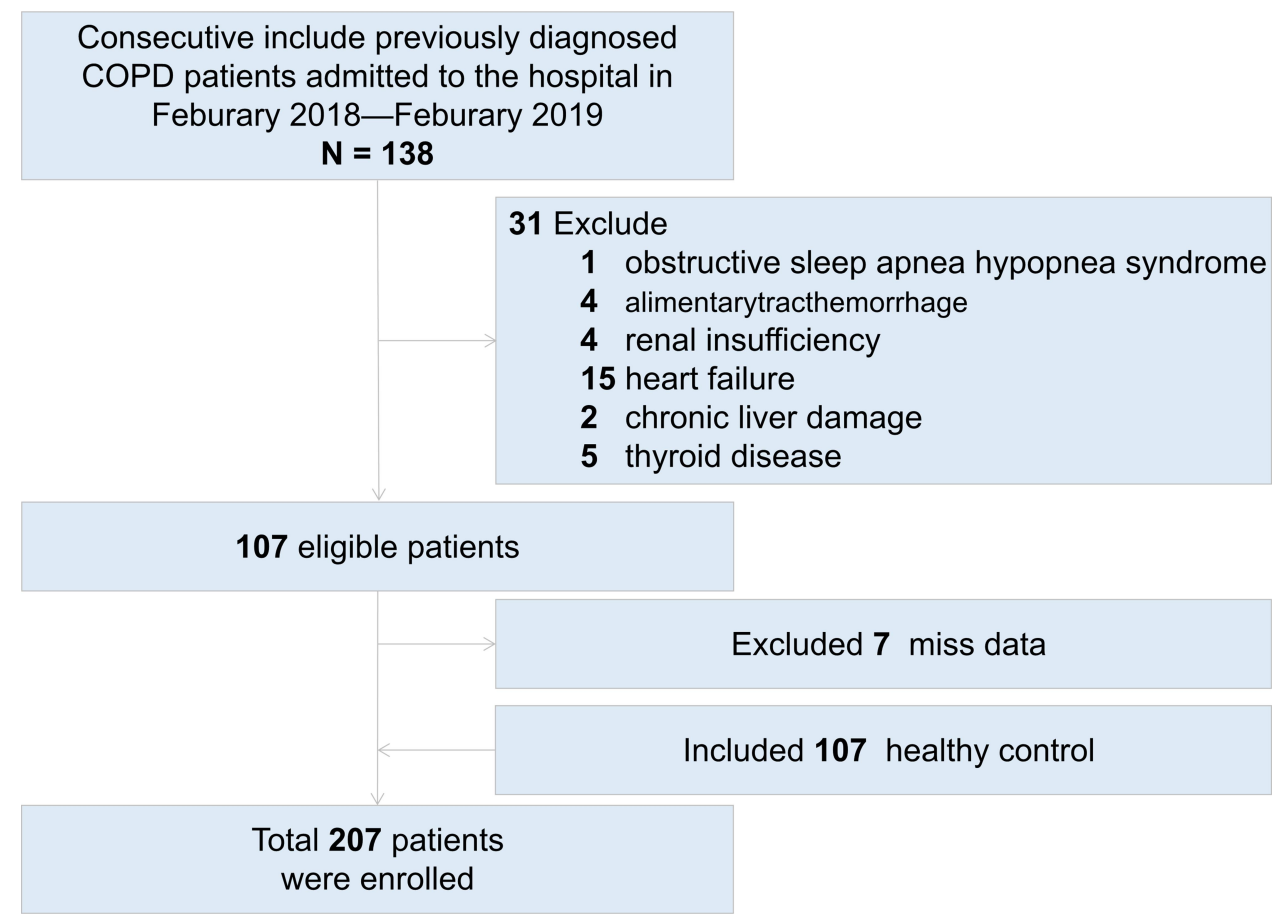

Figure I Flow chart showing the literature search and selection. Specific reasons for exclusion of studies are also shown. Abbreviation: COPD, chronic obstructive pulmonary disease.

existing indicator NLR and PLR, the receiver operating characteristics (ROC) curves were plotted. Besides, De Long method was used to calculate whether there is a significant difference in the area under the curves (AUC). Patients were divided into mild group (GOLD I and GOLD II) and severe group (GOLD III and GOLD IV), as the state variable of ROC test. Multivariate logistic regression analysis was carried out to identify the contribution of the RCI as predictor of severe GOLD stage. Twosided $p$ values $<0.05$ were considered significant in all analyses.

\section{Results}

\section{Baseline Characteristics of the Study Subjects}

Among 207 patients, 100 were COPD patients and another 107 were age and sex matched healthy controls. Compared with the healthy control group, the NLR (4.02 \pm 3.80 vs 1.65 $\pm 0.74, p<0.001)$, PLR $(170.89 \pm 101.61$ vs $99.74 \pm 37.04$, $p<0.001)$, and RCI $(2.10 \pm 1.44$ vs $1.64 \pm 0.64, p=0.004)$ levels in the COPD group were higher (Table 1). NLR and PLR are known as indicators related to the severity of COPD.

In order to gain a further understanding of the correlation between RCI and severity of COPD, 100 COPD patients were divided into two groups according to the ROC cutoff value of $\mathrm{RCI}(\mathrm{RCI}<1.75, \mathrm{n}=54$; $\mathrm{RCI}>1.75, \mathrm{n}=46)$.
As can be seen from Table 2, higher $\mathrm{PaCO}_{2}$ (42.44 [36.97-47.91] vs 46.73 [35.34-58.12], $p=0.025)$ and lower $\mathrm{FEV}_{1}$ $\%$ (44.25 [29.37-59.13] vs 34.10 [21.53-46.67], $p=0.001)$, $\mathrm{FEV}_{1}(1.05$ [0.56-1.54] vs 0.84 [0.5-1.18], $p=0.016)$, FVC (1.97 [1.24-2.7] vs 1.67 [1.13-2.21], $p=0.027)$ were observed among COPD patients with higher RCI. But in $\mathrm{FEV}_{1} / \mathrm{FVC}$ ratio and $\mathrm{PaO}_{2}$, there was no significant difference between the two groups. These results indicate that RCI is related to pulmonary function. Patients with poor pulmonary function and severe COPD had higher RCI. RCI and COPD severity is positively correlated.

Aimed to further explore the clinical significance of RCI, the two groups of low RCI and high RCI were compared from aspects of baseline characteristic and the severity of disease. From Table 2, we can see that there was no obvious difference in age, smoking, hypertension, diabetes mellitus in baseline characteristic, while there were significant differences in gender and duration of disease.

Higher GOLD scale (2.76 [1.92-3.6] vs 3.31 [2.67-3.95], $p=0.001)$, ADO scale (4.41 [3-5.82] vs 5.05 [3.67-6.43], $p=0.033)$ and DOSE scale (2.24 [0.6-3.88] vs (3.21 [1.47-4.95], $p=0.051)$ were observed among patients with higher RCI, but the BODE, SGQR, CAT, mMRC showed no significant differences between the two groups. 
Table I Comparison of COPD Patients and Healthy Controls

\begin{tabular}{|c|c|c|c|c|}
\hline & $\begin{array}{c}\text { Total } \\
(\mathrm{n}=\mathbf{2 0 7})\end{array}$ & $\begin{array}{l}\text { Group A } \\
(n=100)\end{array}$ & $\begin{array}{l}\text { Group B } \\
(n=107)\end{array}$ & $\mathbf{p}$ \\
\hline Age (years) & $70.14 \pm 8.38$ & $70.17 \pm 8.41$ & $70.12 \pm 8.39$ & 0.968 \\
\hline Gender (male, $n \%$ ) & 178 (85.99) & $87(87.00)$ & 91 (85.05) & 0.467 \\
\hline $\operatorname{RBC}\left(10^{12} / \mathrm{L}\right)$ & $4.50 \pm 0.57$ & $4.28 \pm 0.44$ & $4.70 \pm 0.60$ & $<0.001$ \\
\hline $\mathrm{Hb}(\mathrm{g} / \mathrm{L})$ & $137.85 \pm 19.0$ & $130.19 \pm 13.82$ & $145.08 \pm 20.39$ & $<0.001$ \\
\hline PLT $\left(10^{9} / \mathrm{L}\right)$ & $219.84 \pm 65.05$ & $232.75 \pm 75.34$ & $207.66 \pm 51.01$ & 0.006 \\
\hline $\operatorname{Lym}\left(10^{9} / \mathrm{L}\right)$ & $2.61 \pm 9.30$ & $1.62 \pm 0.73$ & $3.54 \pm|2.9|$ & 0.138 \\
\hline $\mathrm{RCl}$ & $1.86 \pm 1.12$ & $2.10 \pm 1.44$ & $1.64 \pm 0.64$ & 0.004 \\
\hline NLR & $2.80 \pm 2.95$ & $4.02 \pm 3.80$ & $1.65 \pm 0.74$ & $<0.001$ \\
\hline PLR & $134.29 \pm 83.42$ & $170.89 \pm 101.61$ & $99.74 \pm 37.04$ & $<0.001$ \\
\hline RDW & $13.12 \pm 1.27$ & $13.27 \pm 0.08$ & $12.98 \pm 0.15$ & 0.055 \\
\hline
\end{tabular}

Notes: Group A, patients with COPD; Group B, healthy people.

Abbreviations: COPD, chronic obstructive pulmonary disease; RBC, red blood cell; Hb, hemoglobin; PLT, platelet; Lym, lymphocyte; RCl, red cell index; NLR, neutrophillymphocyte ratio; PLR, platelet-lymphocyte ratio; RDW, red blood cell distribution width.

Table 2 T-Tests for Groups Divided According to $\mathrm{RCl}$ with Severity of Disease and Other Functions

\begin{tabular}{|c|c|c|c|}
\hline & $\begin{array}{c}\mathrm{RCl}<1.75 \\
(\mathrm{n}=54)\end{array}$ & $\begin{array}{c}\mathrm{RCl}>\mathrm{I} .75 \\
(\mathrm{n}=46)\end{array}$ & $p$ \\
\hline \multicolumn{4}{|l|}{ Baseline characteristic } \\
\hline Age & $69.67 \pm 9.46$ & $71.28 \pm 7.38$ & 0.340 \\
\hline Sex (male, n\%) & $40(74.07)$ & $42(91.30)$ & 0.021 \\
\hline Duration of Disease & $10.13 \pm 8.69$ & $15.69 \pm 13.55$ & 0.021 \\
\hline Smoking & $36(66.67)$ & $38(82.61)$ & 0.054 \\
\hline Hypertension & $22(40.74)$ & $16(34.78)$ & 0.545 \\
\hline Diabetes mellitus & $9(16.67)$ & $6(13.04)$ & 0.617 \\
\hline \multicolumn{4}{|l|}{ Lung Function } \\
\hline $\mathrm{FEV}_{\text {, (L) }}$ & $1.05 \pm 0.49$ & $0.84 \pm 0.34$ & 0.016 \\
\hline FVC (L) & $1.97 \pm 0.73$ & $1.67 \pm 0.54$ & 0.027 \\
\hline $\mathrm{FEV}_{1} \%(<50 \%)$ & $44.25 \pm 14.88$ & $34.10 \pm 12.57$ & 0.001 \\
\hline $\mathrm{FEV}_{\mathrm{I}} / \mathrm{FVC}$ ratio (\%) & $52.16 \pm 9.63$ & $49.46 \pm 7.69$ & 0.150 \\
\hline $\mathrm{PaO}_{2}$ & $73.21 \pm 12.33$ & $72.57 \pm 17.47$ & 0.838 \\
\hline $\mathrm{PaCO}_{2}$ & $42.44 \pm 5.47$ & $46.73 \pm 11.39$ & 0.025 \\
\hline \multicolumn{4}{|l|}{ Severity of disease } \\
\hline GOLD & $2.76 \pm 0.84$ & $3.31 \pm 0.64$ & 0.001 \\
\hline BODE & $3.56 \pm 2.22$ & $4.25 \pm 2.45$ & 0.165 \\
\hline ADO & $4.41 \pm 1.41$ & $5.05 \pm 1.38$ & 0.033 \\
\hline DOSE & $2.24 \pm 1.64$ & $3.21 \pm 1.74$ & 0.051 \\
\hline $\mathrm{mMRC}$ & $1.58 \pm 0.97$ & $1.77 \pm 0.96$ & 0.343 \\
\hline CAT & $17.45 \pm 6.64$ & $18.18 \pm 6.91$ & 0.601 \\
\hline SGQR & $36.30 \pm 19.82$ & $39.42 \pm 2.55$ & 0.415 \\
\hline
\end{tabular}

Abbreviations: $\mathrm{RCl}$, red cell index; $\mathrm{FEV}_{\mathrm{l}}$, forced expiratory volume in I second; $\mathrm{FVC}$, forced vital capacity; $\mathrm{FEV}, \%$, forced expiratory volume in I second in percent of the predicted value; $\mathrm{PaO}_{2}$, partial pressure of oxygen in arterial blood; $\mathrm{PaCO}_{2}$, partial pressure of carbon dioxide in arterial blood; GOLD, Global Initiative for Chronic Obstructive Lung Disease; BODE, BMI, airflow obstruction, dyspnea and exercise capacity; ADO, age, dyspnoea and obstruction; DOSE, dyspnoea, obstruction, smoking and exacerbations; mMRC, modified Medical Research Council dyspnoea scale; CAT, COPD assessment test; SGRQ, St. George's Respiratory Questionnaire.

\section{Comparisons of the RCI, NLR and PLR in COPD}

The Pearson's correlation indicated that there was a significant negative correlation between RCI and $\mathrm{FEV}_{1}$ $\%(r=-0.302, p=0.004)$, while there was no correlation between NLR $(r=-0.153, p=0.148)$, PLR $(r=-0.098$, $p=0.354$ ) and $\mathrm{FEV}_{1} \%$ (Figure 2). Afterwards, the ROC curve analysis was performed to evaluate the utilities of RCI, NLR and PLR for predicting COPD severity level, here referred to GOLD. The analysis showed that the AUC values of NLR, PLR, Lym, Red blood cell distribution width (RDW) were $0.654 \quad(95 \%$ CI: $0.54-0.77$, $p=0.020), 0.611$ (95\% CI: 0.49-0.74, $p=0.095), 0.705$ (95\% CI: $0.178-0.412, p=0.002)$ and $0.515(95 \% \mathrm{CI}$ : $0.382-0.649, p=0.819)$. On the contrary, at a cut-off value of 1.75 , the specificity and sensitivity of RCI in predicting lung function were $57.6 \%$ and $85.2 \%$, with an AUC of 0.729 (95\% CI: $0.62-0.84, p=0.001$ ) (Figure 3). It indicates that RCI 's AUC is significantly larger than the indicators related to the severity of COPD that have been proven such as NLR, PLR and RDW.

\section{$\mathrm{RCl}$ and Pulmonary Function are Closely Related}

In low RCI group, 53.7\% suffered from poor pulmonary function $\left(\mathrm{FEV}_{1} \%<50\right)$, while the proportion increased to $78.3 \%$ in high RCI group (Table 2), which indicates that the higher the RCI, the lower the $\mathrm{FEV}_{1} \%$ and the worse 

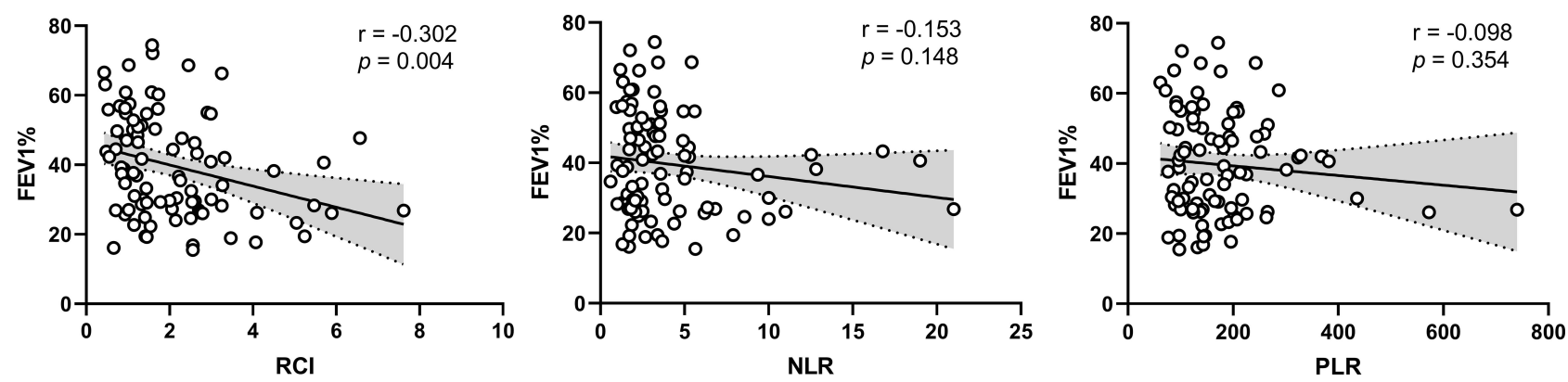

Figure 2 Correlations of the RCl, NLR and PLR with $\mathrm{FEV}, \%$.

Notes: Correlations between RCl, NLR and PLR levels in COPD patients and FEV $\%$ were assessed by Pearson's correlation test, RCl, $r=-0.302, p=0.004 ; \mathrm{NLR}, r$ $=-0.153, p=0.148$; and PLR, $r=-0.098, p=0.354$.

the pulmonary function, manifesting $\mathrm{RCI}$ and $\mathrm{FEV}_{1} \%$ is inversely proportional.

To further explore the independent factors of pulmonary function, variables were subjected to univariate logistic regression analyses. Hence, duration of disease $(p=0.031)$, $\operatorname{BMI}(p=0.020), \mathrm{FEV}_{1} / \mathrm{FVC}(p<0.001), \mathrm{PaCO}_{2}(p<0.001)$ and RCI $(p=0.003)$ were observed to have a significant correlation with severe COPD (Mild: GOLD I, GOLD II; Severe: GOLD III, GOLD IV) (Table 3).

To control other potential confounding variables, multivariate logistic regression analyses were performed. In Model 1, nothing was adjusted (odds ratio $[\mathrm{OR}]=2.53$,

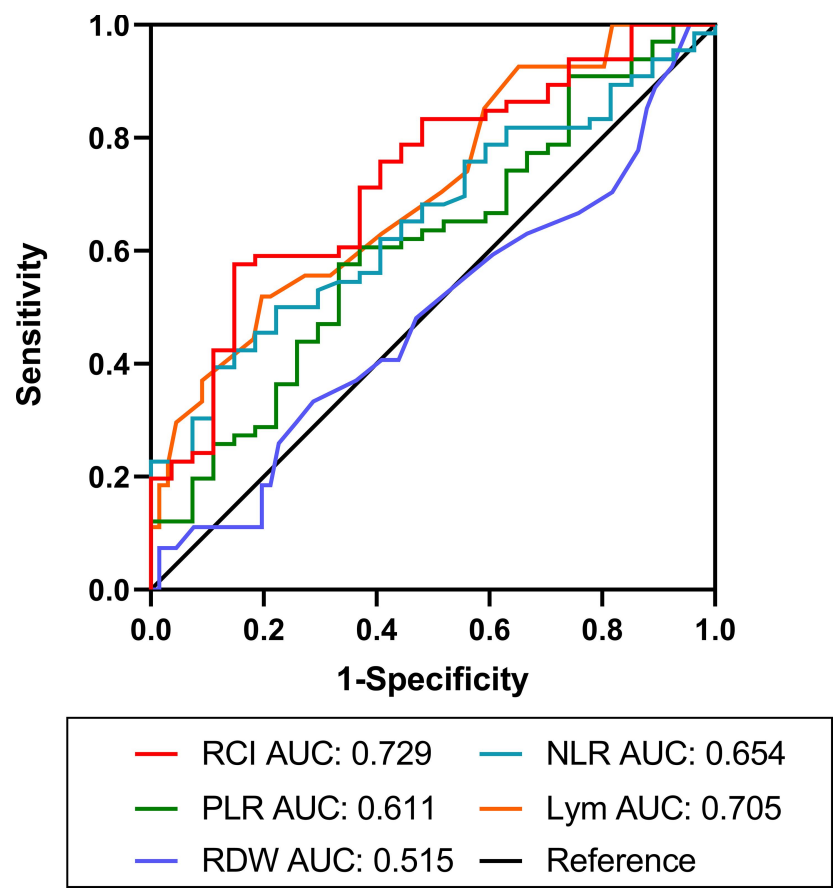

Figure 3 ROC curves of the RCI, NLR, PLR, Lym and RDW of COPD patients, for predicting severe or mild COPD. The area under ROC curve of RCl: 0.729 ; $95 \%$ Cl: $0.619-0.839 ; p=0.001$.
95\% CI:1.38-4.66, $p=0.003$ ). After adjusted for age, sex, BMI, and duration of disease in Model 2, the linkage between $\mathrm{RCI}$ and GOLD remained significant $([\mathrm{OR}]=2.35,95 \% \mathrm{CI}$ : $1.24-4.45, p=0.009)$. On the basis of Model 2, we additionally made adjustments for $\mathrm{FEV}_{1} / \mathrm{FVC}$, and $\mathrm{PaCO}_{2}$ in Model 3 , the linkage between RCI and GOLD still remained significant $([\mathrm{OR}]=4.27,95 \% \mathrm{CI}: 1.57-11.63, p=0.004)$. After adjusted for potential confounders, there was still a correlation between RCI and GOLD, which showed that $\mathrm{RCI}$ is probably an independent impact factor of COPD severity (Table 4).

\section{Discussion}

Our data showed that COPD patients had higher RCI levels than healthy controls. Besides, higher RCI levels were associated with poor pulmonary function and severe COPD. In addition, RCI has higher predictive value than NLR, PLR and RDW for COPD severity. RCI is obtained by calculating $(\mathrm{RBC} \times \mathrm{Hb}) /(\mathrm{Lym} \times \mathrm{PLT})$ which is closely related to the four indicators of $\mathrm{RBC}, \mathrm{Hb}$, Lym and PLT. Some previous studies and possible explanations are shown below.

In this study, we found that the lymphocyte count of COPD patients was lower than that of healthy controls. Acanfora's study found similar results that a low relative lymphocyte count was associated with a high mortality rate in elderly patients with severe COPD. ${ }^{27}$ Other studies have also shown that when patients were at a severe disease level, they have a decreased lymphocyte count compared to healthy controls or patients in the stable phase. ${ }^{28}$ The mechanism of lymphocyte influence on COPD can be explained by a characteristic cellular immune response. This immunopathology is driven by lymphocytes and responded to the targeted immune regulation of human lymphocytes. Besides, when the lymphocyte count is less 
Table 3 Univariate Logistic Regression Analysis Assesses Different Prognostic Predictors of COPD

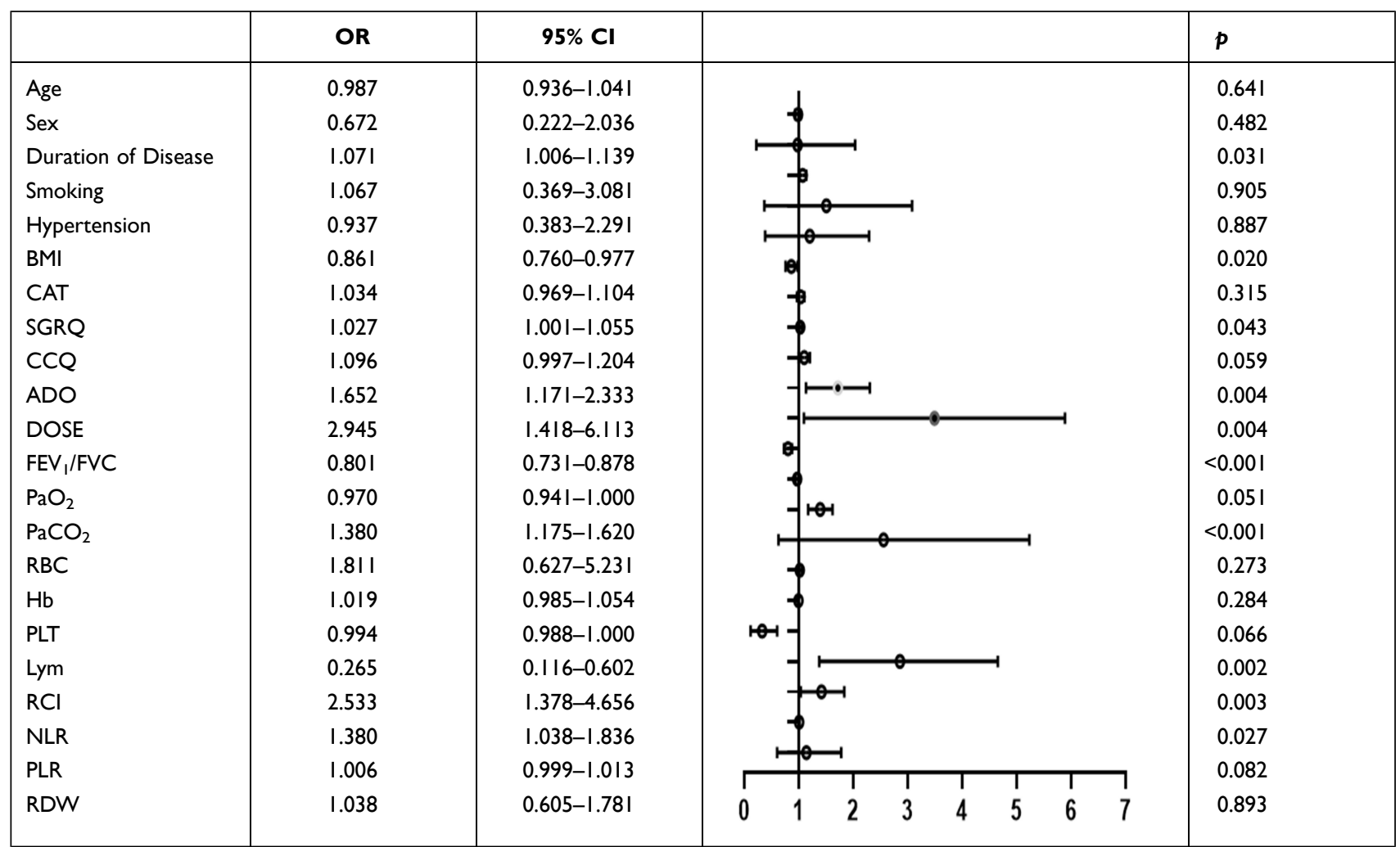

Notes: Divided COPD patients into two groups according to GOLD stage: Group A, GOLD I + GOLD II; Group B, GOLD III + GOLD IV. Using univariate logistic regression analysis to assess the effect of prognostic indicators on the severity of COPD.

Abbreviations: BMI, body mass index; CAT, COPD assessment test; SGRQ, St. George's Respiratory Questionnaire; CCQ, Clinical COPD Questionary; ADO, age, dyspnea and airflow obstruction; DOSE, MRC Dyspnea Scale, airflow obstruction, smoking status and exacerbations; FEV forced vital capacity; $\mathrm{PaO}_{2}$, partial pressure of oxygen in arterial blood; $\mathrm{PaCO}_{2}$, partial pressure of carbon dioxide in arterial blood; RBC, red blood count; $\mathrm{Hb}$, hemoglobin; PLT, platelet; Lym, lymphocyte; RCl, red cell index; NLR, neutrophil-lymphocyte ratio; PLR, platelet-lymphocyte ratio; RDW, red blood cell distribution width.

than 1500, it often indicates malnutrition and the relationship between the severity of COPD and malnutrition has been explained by Collins PF et al. ${ }^{29}$

Our study found that $\mathrm{RBC}$ and $\mathrm{Hb}$ are significantly lower in COPD patients than that of healthy controls. It is known that the increase in $\mathrm{RBC}$ and $\mathrm{Hb}$ levels reflected the sensitivity to hypoxia ${ }^{30,31}$ and increased levels of RBC and $\mathrm{Hb}$ can compensate for poor pulmonary function. ${ }^{32,33}$ The possible mechanisms are listed below.

Table 4 Adjusted Odds Ratio (95\% Confidence Interval) for Severe COPD (GOLD III + IV)

\begin{tabular}{|l|c|c|}
\hline & OR $(\mathbf{9 5 \%} \mathbf{~ C l})$ & $\boldsymbol{P}$ \\
\hline Modell & $2.533(1.378-4.656)$ & 0.003 \\
Model2 & $2.348(1.239-4.450)$ & 0.009 \\
Model3 & $4.272(1.570-11.627)$ & 0.004 \\
\hline
\end{tabular}

Notes: Model I is univariate analysis. Model 2 is adjusted by age, sex, duration of disease and body mass index. Model 3 is adjusted by age, sex, duration of disease, $\mathrm{BMI}, \mathrm{FEV} / \mathrm{FVC}$ and $\mathrm{PaCO}_{2}$.
1) Anemia. Anemia is defined as $\mathrm{Hb}<12.0 \mathrm{~g} / \mathrm{dL}$ for males, and $<11.0 \mathrm{~g} / \mathrm{dL}$ for females. ${ }^{10}$

In adults with COPD, anemia is associated with worse exercise capacity, greater breathing difficulties, and more serious diseases. In addition, studies have shown that anemia is associated with an increasing in the long-term mortality of COPD, and even mild anemia is also associated with a significant increase in risk. ${ }^{34}$ In patients with acute exacerbations of COPD, anemia may be a risk factor of hospital death for patients with severe COPD who need mechanical ventilation support. ${ }^{35}$

2) Red blood cell oxidative modification. Red blood cell oxidative modification is a valuable biological indicator in the clinical treatment of COPD. ${ }^{30}$ This study proved that in COPD patients PLT is significantly higher than that of healthy controls. ${ }^{17}$ Potential mechanism can be explained by systemic inflammation. COPD is related to systemic inflammation and activated platelets can recruit inflammatory cells to make them aggregate. The interaction between platelets and inflammatory cells can 
stimulate the release of chemokines and the further recruitment of immune substances. ${ }^{36}$

$\mathrm{RBC}$ and $\mathrm{Hb}$ can be used as appropriate criteria for evaluating pulmonary function, under the premise of excluding the influence of other factors on the changes in red blood cell proliferation on $\mathrm{RBC}$ and $\mathrm{Hb}$ levels. Lym and PLT are rarely affected by other factors, so they are benchmarks used to measure the total premeability of blood cell. The red blood cell index obtained from this can accurately reflect the degree of compensation. When the body has respiratory failure, it is often in a poor state, so the general blood cell proliferation level may be abnormal. Hence, it is necessary to determine a variable accurately which can reflects the changes in blood cell proliferation levels. RCI is considered to reflect the compensatory increase in $\mathrm{RBC}$ count and $\mathrm{Hb}$ level, secondary to poor lung function which can also reflect the true state of pulmonary function. ${ }^{13}$

Other research results showed that the NLR of COPD patients in the stable phase is significantly higher than that of the healthy control group. And compared with the stable phase, it also increased as the disease aggravated. Therefore, NLR is a fast, cheap, and easy-to-measure indicator that can be used for routine whole blood technical analysis. ${ }^{37}$ The same conclusion was also drawn in the meta-analysis conducted by Paliogiannis et al. ${ }^{38}$ In our research, there was no correlation between lung function and NLR (Figure 2). However, researchers have made conclusion opposite to ours'. ${ }^{39}$ This may be due to the different sample size. We have 100 cases and their sample size is much larger than ours.

As for PLR, the PLR of patients with stable COPD was significantly higher than that of healthy controls, while the PLR level of non-survivors with acute exacerbation of COPD is significantly higher, but compared with NLR, PLR is still a more effective and simple prognostic indicators. ${ }^{40}$ The crosssectional multi-center study of Alexa et $\mathrm{al}^{7}$ showed that NLR and PLR are predictors of COPD, while our results illustrated there were no correlations between them and $\mathrm{FEV}_{1} \%$. Among GODL A-D group, no differences of NLR and PLR were found either. ${ }^{7}$ Additionally, our results elucidated that RCI was significantly negatively related to $\operatorname{FEV}_{1}(p=0.016)$, $\operatorname{FEV}_{1} \%(p=0.001)$ and FVC $(p=0.027)$ (Table 2$)$, while no correlations between NLR $(p=0.148)$ or $\operatorname{PLR}(p=0.354)$ and pulmonary function were found (Figure 2).

Previous work has explored red blood cell distribution and its predictive value of COPD, which proved that RDW was related to the severity of COPD. However, in our research, there was no significant difference between COPD patients and healthy controls (Table 1). And the logistic analysis showed no correlation between RDW and GOLD level, which reflects disease severity (Table 3). This difference may be related to our small simple size. Some researchers found same conclusion. Gunay et al found that in stable COPD patients, there was no correlation with RDW and GOLD level. ${ }^{41}$

Our article is the first to use RCI as a predictive biomarker for the severity of COPD. Previously, RCI was only used as an effective index for evaluating pulmonary function with complete blood count parameters. Compared with the healthy control group, the elderly COPD group had high RCI, and the positive rate of abnormally elevated $\mathrm{RCI}$ in the COPD group and the elderly group were significantly higher than that of the control group. ${ }^{13}$

However, our study also has certain limitations. Firstly, the sample size is relatively small. As shown in Table 1, our population is heavily skewed towards male gender, which may affect the effectiveness of RCI as an indicator of the severity of COPD in female patients.

Previous research has found that there were significant differences between male patients and female patients in terms of disease severity, comorbidities and prognosis. ${ }^{42,43}$ Although COPD has always been considered as the disease of elderly men, the burden of COPD on female patients has gradually increased in recent years. We hope that there will be more studies on female COPD patients in the future. Secondly, we are a single-center cross-sectional survey that only focuses on the Chinese population. Finally, RCI was only used in COPD patients in China for now. In this regard, we need to conduct prospective cohort studies and research on different ethnic groups, recruit more volunteers from multiple centers, and further explore the mechanism of RCI in the progress of COPD to solve the problems mentioned above.

\section{Conclusion}

In summary, RCI is a novel biomarker that can better assess the pulmonary function and severity of COPD than NLR and PLR. Increased RCI is independently related to deterioration of lung pulmonary function.

\section{Abbreviations}

COPD, chronic obstructive pulmonary disease; RCI, red cell index; NLR, neutrophil-lymphocyte ratio; PLR, platelet-lymphocyte ratio; $\mathrm{Hb}$, Hemoglobin; Lym, Lymphocyte; PLT, Platelet; $\mathrm{FEV}_{1} \%$, forced expiratory volume in 1 second in percent of the predicted value; 
$\mathrm{FEV}_{1}$, forced expiratory volume in 1 second; FVC, forced vital capacity; BODE, body mass index, airway obstruction, dyspnoea, exercise capacity; SGRQ, St. George's Respiratory Questionnaire; GOLD, Global Initiative for Chronic Obstructive Lung Disease; ADO, age, dyspnea and airflow obstruction; DOSE, MRC Dyspnea Scale, airflow obstruction, smoking status and exacerbations; $\mathrm{BMI}$, body mass index; $\mathrm{PaCO}_{2}$, partial pressure of carbon dioxide in arterial blood; $\mathrm{PaO}_{2}$, partial pressure of oxygen in arterial blood; $\mathrm{ROC}$, receiver operating characteristics; AUC, the area under the curve.

\section{Data Sharing Statement}

The datasets generated during and/or analysed during the current study are available from the corresponding author on reasonable request.

\section{Ethics Approval and Informed Consent}

All participants provided written informed consent for participation in this study. The study was conducted in accordance with the ethical standards of the Ethics Committees of the Third Affiliated Hospital of Wenzhou Medical University (YJ20170015).

\section{Acknowledgments}

We thank all the participants in the study and the staff at the Third Affiliated Hospital of Wenzhou Medical University for their contribution in obtaining the data.

\section{Author Contributions}

Conceptualization: Xiaodiao Zhang, Meiying Cao; Methodology: Xiaodiao Zhang, Meiying Cao, Yiben Huang, Jianing Wang; Formal analysis: Jianing Wang, Jiamin Shen, Jiedong Ma, Xiaqi Miao; Investigation: Xiaodiao Zhang, Meiying Cao, Yiben Huang; Writing original draft preparation: Yiben Huang, Jiang Wang, Jiamin Shen; Writing - review and editing: Xiaodiao Zhang, Meiying Cao; Methodology: Xiaodiao Zhang, Meiying Cao, Yiben Huang, Jianing Wang, Jiamin Shen, Jiedong Ma, Xiaqi Miao, Keke Ding, Bingqian Jiang, Binbin Hu, Fangyi Fu, Lingzhi Huang; Funding acquisition: Xiaodiao Zhang, Meiying Cao; Resources: Xiaodiao Zhang, Meiying Cao; Supervision: Xiaodiao Zhang, Meiying Cao. All authors made substantial contributions to conception and design, acquisition of data, or analysis and interpretation of data; took part in drafting the article or revising it critically for important intellectual content; agreed to submit to the current journal; gave final approval of the version to be published; and agree to be accountable for all aspects of the work.

\section{Funding}

This work was funded by the Wenzhou Municipal SciTech Bureau Program (grant number Y20180365). The Funding body had no role in the design of the study, data collection, interpretation of data, statistical analysis or the draft of the manuscript.

\section{Disclosure}

The authors declare that they have no competing interests in this work.

\section{References}

1. Lopez-Campos JL, Tan W, Soriano JB. Global burden of COPD. Respirology. 2016;21(1):14-23. doi:10.1111/resp.12660

2. Rabe KF, Watz H. Chronic obstructive pulmonary disease. The Lancet. 2017;389(10082):1931-1940. doi:10.1016/s0140-6736(17)31222-9

3. Eisner M, Blanc P, Sidney S, et al. Body composition and functional limitation in COPD. Respir Res. 2007;8:7. doi:10.1186/ 1465-9921-8-7

4. Andelid K, Bake B, Rak S, Linden A, Rosengren A, Ekberg-Jansson A. Myeloperoxidase as a marker of increasing systemic inflammation in smokers without severe airway symptoms. Respir Med. 2007;101 (5):888-895. doi:10.1016/j.rmed.2006.09.023

5. Houghton AM, Rzymkiewicz DM, Ji H, et al. Neutrophil elastase-mediated degradation of IRS-1 accelerates lung tumor growth. Nat Med. 2010;16(2):219-223. doi:10.1038/nm.2084

6. El-Gazzar A, Kamel M, Elbahnasy O, El-Naggar MJ. Prognostic value of platelet and neutrophil to lymphocyte ratio in COPD patients. 2020;14(1):111-116. doi:10.1080/17476348.2019.1675517

7. Nunez A, Marras V, Harlander M, et al. Association between routine blood biomarkers and clinical phenotypes and exacerbations in chronic obstructive pulmonary disease. Int $J$ Chron Obstruct Pulmon Dis. 2020;15:681-690. doi:10.2147/COPD.S240720

8. Skoczynski S, Krzyzak D, Studnicka A, et al. Chronic obstructive pulmonary disease and platelet count. Adv Exp Med Biol. 2019;1160:19-23. doi:10.1007/5584_2019_379

9. Makhlouf H, Sadek S, Nafady AJT. Platelet function in diabetic and nondiabetic patients with chronic obstructive pulmonary disease: a case control study. Clin Respir J. 2018;12(1):48-56. doi:10.1111/crj.12477

10. Guo J, Zheng C, Xiao Q, et al. Impact of anaemia on lung function and exercise capacity in patients with stable severe chronic obstructive pulmonary disease. BMJ Open. 2015;5(10):e008295. doi:10.1136/bmjopen-2015-008295

11. Rasmussen L, Christensen S, Lenler-Petersen P, Johnsen SP. Anemia and 90-day mortality in COPD patients requiring invasive mechanical ventilation. Clin Epidemiol. 2010;3:1-5. doi:10.2147/ CLEP.S12885

12. Gadre SK, Jhand AS, Abuqayyas S, Wang X, Guzman J, Duggal A. Effect of anemia on mortality in mechanically ventilated patients with Chronic Obstructive Pulmonary Disease. J Intensive Care Med. 2020;35(3):251-256. doi:10.1177/0885066617739561

13. Guang Y, Jie Z, Feng D, Hui L. Surrogate scale for evaluating respiratory function based on complete blood count parameters. J Clin Lab Anal. 2018;32(5):e22385. doi:10.1002/jcla.22385 
14. Vogelmeier C, Criner G, Martinez F, et al. Global strategy for the diagnosis, management, and prevention of chronic obstructive lung disease 2017 report. GOLD Executive Summary. 2017;195 (5):557-582. doi:10.1164/rccm.201701-0218PP

15. Guang Y, Jie Z, Feng D, Hui LJ. Surrogate scale for evaluating respiratory function based on complete blood count parameters. J Clin Lab Anal. 2018;32(5):e22385. doi:10.1002/jcla.22385

16. Şahin F, Koşar A, Aslan A, Yiğitbaş B, Uslu BJJ. Serum biomarkers in patients with stable and acute exacerbation of chronic obstructive pulmonary disease: a comparative study. J Med Biochem. 2019;38 (4):503-511. doi:10.2478/jomb-2018-0050

17. Skoczyński S, Krzyżak D, Studnicka A, et al. Chronic obstructive pulmonary disease and platelet count. Advan Exp Med Biol. 2019;1160:19-23. doi:10.1007/5584_2019_379

18. García-Rivero J, Esquinas C, Barrecheguren M, et al. Risk factors of poor outcomes after admission for a COPD exacerbation: multivariate logistic predictive models. COPD. 2017;14(2):164-169. doi:10.1080/15412555.2016.1260538

19. JAjor and medicine, cc. ATS/ERS Statement on respiratory muscle testing. Am J Respir Crit Care Med. 2002;166(4):518-624. doi:10.1164/rccm.166.4.518

20. Vaz Fragoso CA, Concato J, McAvay G, et al. The ratio of FEV1 to FVC as a basis for establishing chronic obstructive pulmonary disease. Am J Respir Crit Care Med. 2010;181(5):446-451. doi:10.1164/rccm.200909-1366OC

21. Agarwala P, Salzman SJC. Six-minute walk test: clinical role, technique, coding, and reimbursement. Chest. 2020;157(3):603-611 doi:10.1016/j.chest.2019.10.014

22. Chu J, Chen X, Shen S, et al. A poor performance in comprehensive geriatric assessment is associated with increased fall risk in elders with hypertension: a cross-sectional study. $J$ Geriatric Cardiol. 2015;12(2):113-118. doi:10.11909/j.issn.1671-5411.2015.02.006

23. Celli B, Cote C, Marin J, et al. The body-mass index, airflow obstruction, dyspnea, and exercise capacity index in chronic obstructive pulmonary disease. $N$ Engl J Med. 2004;350(10):1005-1012. doi:10.1056/NEJMoa021322

24. Puhan M, Garcia-Aymerich J, Frey M, et al. Expansion of the prognostic assessment of patients with chronic obstructive pulmonary disease: the updated BODE index and the ADO index. Lancet (London, England). 2009;374(9691):704-711. doi:10.1016/s01406736(09)61301-5

25. Jones R, Donaldson G, Chavannes N, et al. Derivation and validation of a composite index of severity in chronic obstructive pulmonary disease: the DOSE Index. Am J Respir Crit Care Med. 2009;180 (12):1189-1195. doi:10.1164/rccm.200902-02710C

26. Vestbo J, Hurd SS, Agusti AG, et al. Global strategy for the diagnosis, management, and prevention of chronic obstructive pulmonary disease: GOLD executive summary. Am J Respir Crit Care Med. 2013;187(4):347-365. doi:10.1164/rccm.201204-0596PP

27. Acanfora D, Scicchitano P, Carone M, et al. Relative lymphocyte count as an indicator of 3-year mortality in elderly people with severe COPD. BMC Pulm Med. 2018;18(1):116. doi:10.1186/s12890-0180685-6

28. Şahin F, Koşar AF, Aslan AF, Yiğitbaş B, Uslu B. Serum biomarkers in patients with stable and acute exacerbation of chronic obstructive pulmonary disease: a comparative study. J Med Biochem. 2019;38 (4):503-511. doi:10.2478/jomb-2018-0050
29. Collins PF, Elia M, Kurukulaaratchy RJ, Stratton RJ. The influence of deprivation on malnutrition risk in outpatients with chronic obstructive pulmonary disease (COPD). Clin Nutr. 2018;37(1):144-148. doi:10.1016/j.clnu.2016.11.005

30. Casanova C, Marin J, Martinez-Gonzalez C, et al. Differential effect of modified medical research council dyspnea, COPD assessment test, and clinical COPD questionnaire for symptoms evaluation within the new GOLD Staging and Mortality in COPD. Chest. 2015;148(1):159-168. doi:10.1378/chest.14-2449

31. Samaja M, Crespi T, Guazzi M, Vandegriff KD. Oxygen transport in blood at high altitude: role of the hemoglobin-oxygen affinity and impact of the phenomena related to hemoglobin allosterism and red cell function. Eur $J$ Appl Physiol. 2003;90(3-4):351-359. doi:10.1007/s00421-003-0954-8

32. Gong H, Linn WS, Terrell SL, et al. Exposures of elderly volunteers with and without chronic obstructive pulmonary disease (COPD) to concentrated ambient fine particulate pollution. Inhal Toxicol. 2004;16(11-12):731-744. doi:10.1080/08958370490499906

33. Santos MC, Oliveira AL, Viegas-Crespo AM, et al. Systemic markers of the redox balance in chronic obstructive pulmonary disease. Biomarkers. 2004;9(6):461-469. doi:10.1080/13547500400024768

34. Park SC, Kim YS, Kang YA, et al. Hemoglobin and mortality in patients with COPD: a nationwide population-based cohort study. Int J Chron Obstruct Pulmon Dis. 2018;13:1599-1605. doi:10.2147/COPD.S159249

35. Ergan B, Ergun R. Impact of anemia on short-term survival in severe COPD exacerbations: a cohort study. Int J Chron Obstruct Pulmon Dis. 2016;11:1775-1783. doi:10.2147/COPD.S111758

36. Maclay JD, McAllister DA, Johnston S, et al. Increased platelet activation in patients with stable and acute exacerbation of COPD. Thorax. 2011;66(9):769-774. doi:10.1136/thx.2010.157529

37. Gunay E, Sarinc Ulasli S, Akar O, et al. Neutrophil-to-lymphocyte ratio in chronic obstructive pulmonary disease: a retrospective study. Inflammation. 2014;37(2):374-380. doi:10.1007/s10753-013-9749-1

38. Paliogiannis P, Fois AG, Sotgia S, et al. The neutrophil-tolymphocyte ratio as a marker of chronic obstructive pulmonary disease and its exacerbations: a systematic review and meta-analysis. Eur J Clin Invest. 2018;48(8):e12984. doi:10.1111/eci.12984

39. Gao X, Coull B, Lin X, et al. Association of neutrophil to lymphocyte ratio with pulmonary function in a 30-year longitudinal study of US veterans. 2020;3(7):e2010350. doi:10.1001/jamanetworkopen. 2020.10350

40. Yao C, Liu X, Tang Z. Prognostic role of neutrophil-lymphocyte ratio and platelet-lymphocyte ratio for hospital mortality in patients with AECOPD. Int J Chron Obstruct Pulmon Dis. 2017;12:2285-2290. doi:10.2147/COPD.S141760

41. Günay E, Sarınç Ulaşlı S, Akar O, et al. Neutrophil-to-lymphocyte ratio in chronic obstructive pulmonary disease: a retrospective study. Inflammation. 2014;37(2):374-380. doi:10.1007/s10753-013-9749-1

42. Grabicki M, Kuźnar-Kamińska B, Rubinsztajn R, et al. COPD course and comorbidities: are there gender differences? Advan Exp Med Biol. 2019;1113:43-51. doi:10.1007/5584_2018_160

43. Ntritsos G, Franek J, Belbasis L, et al. Gender-specific estimates of COPD prevalence: a systematic review and meta-analysis. Int J Chronic Obstructive Pulmonary Dis. 2018;13:1507-1514. doi:10.2147/copd.S146390 


\section{Publish your work in this journal}

The International Journal of COPD is an international, peer-reviewed journal of therapeutics and pharmacology focusing on concise rapid reporting of clinical studies and reviews in COPD. Special focus is given to the pathophysiological processes underlying the disease, intervention programs, patient focused education, and self management protocols. This journal is indexed on PubMed Central, MedLine and CAS. The manuscript management system is completely online and includes a very quick and fair peer-review system, which is all easy to use. Visit http://www.dovepress.com/testimonials.php to read real quotes from published authors. 